Supporting information

S1 Appendix. Sample Design and Data Collection Process

\begin{tabular}{|c|c|c|}
\hline \multirow[t]{2}{*}{ Sample design } & Landline & $\begin{array}{l}\text { - Phase 1: the municipality. Stratified randomized selection using the size of the } \\
\text { municipality and the region. } \\
\text { - Phase 2: household. Randomized selection using the Irismedia directory recoded } \\
\text { and debugged by IMOP. } \\
\text { - Phase 3: individual. Selection employing sex and age quotas. } \\
\text { The application selects the household member who is relatively less represented in } \\
\text { the sample at the time of the call and establishes a postponement if the chosen person } \\
\text { is not at home at that moment. }\end{array}$ \\
\hline & $\begin{array}{c}\text { Mobile } \\
\text { phone }\end{array}$ & $\begin{array}{l}\text { Simple random selection using the mobile phone database generated by IMOP from } \\
\text { the data provided by each mobile operator. This database was tested before beginning } \\
\text { the survey in order to detect inactive lines. }\end{array}$ \\
\hline \multicolumn{2}{|l|}{ Technique } & All the interviews are conducted through the CATI system using a computer. \\
\hline \multicolumn{2}{|l|}{ Sample error } & $\pm 1.8 \%$ for a confidence level of $95.5 \%$ \\
\hline \multicolumn{2}{|c|}{$\%$ mobile phone interviews } & $40.4 \%$ \\
\hline \multicolumn{2}{|c|}{$\%$ landline (fixed phone) interviews } & $59.6 \%$ \\
\hline \multicolumn{2}{|c|}{ Questionnaire duration (on average) } & 21.5 minutes \\
\hline \multicolumn{2}{|c|}{ Denial rate } & $14.7 \%$ of the people who took the telephone call declined to answer the questionnaire. \\
\hline \multicolumn{2}{|l|}{ Not completion } & $\begin{array}{l}0.9 \% \text { of the people who began answering the questionnaire decided to end the survey } \\
\text { before its completion. }\end{array}$ \\
\hline \multicolumn{2}{|l|}{ Data availability } & $\begin{array}{l}\text { All data are available at Funcas Foundation (Caballero de Gracia, } 2828013 \text { Madrid). } \\
\text { Contact details: odf@ funcas.es }\end{array}$ \\
\hline
\end{tabular}

S2 Appendix. List of Survey Questionnaire Variables

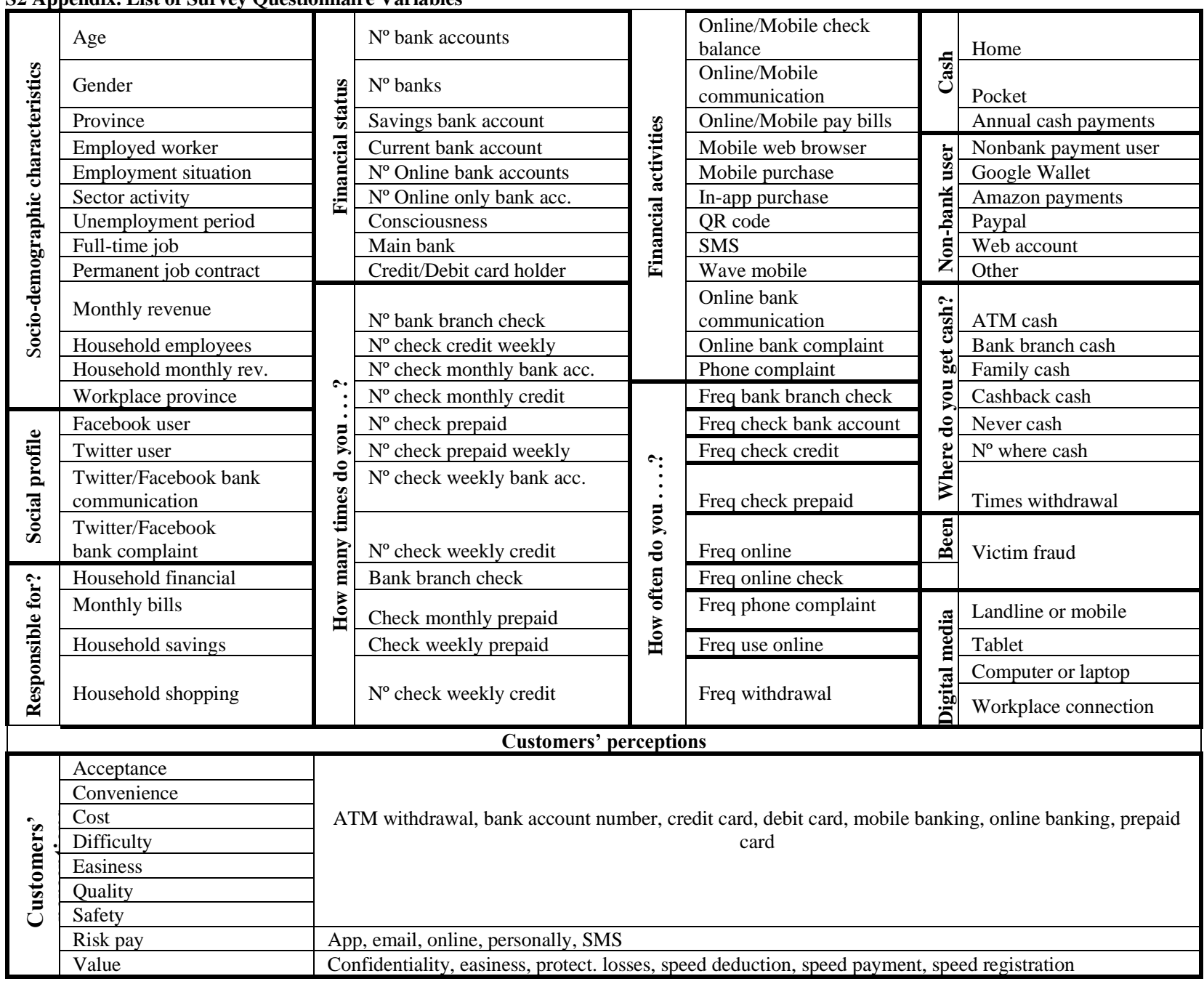


S3 Appendix. Dictionary

\begin{tabular}{|c|c|c|}
\hline Dimension & Classification & Definition \\
\hline \multirow{3}{*}{$\begin{array}{l}\text { Adoption of Digital } \\
\text { Banking }\end{array}$} & Non users & $\begin{array}{l}\text { Individuals who over the course of the year have not adopted any kind of financial } \\
\text { digitalization, including those who are not even digitalized consumers (i.e., they do not use the } \\
\text { internet) }\end{array}$ \\
\hline & Occasional users & Individuals who conducted online banking activities, but not on a monthly basis. \\
\hline & Frequent users & Individuals who conducted online financial activities every month over the course of the year. \\
\hline \multirow{4}{*}{$\begin{array}{l}\text { Degree of use of Digital } \\
\text { Banking }\end{array}$} & No digital users & $\begin{array}{l}\text { Individuals who are outside of the digitalization process (i.e., who have no access to the } \\
\text { internet) }\end{array}$ \\
\hline & $\begin{array}{l}\text { Non-users of digital } \\
\text { financial services }\end{array}$ & Individuals who are frequent internet users but do not conduct any financial activity online \\
\hline & Incipient users & Individuals who perform some but not all online financial activities at least once a month. \\
\hline & Diversified users & Individuals that carry out all financial activities online at least once a month. \\
\hline \multirow{4}{*}{$\begin{array}{l}\text { Adoption of Banks' } \\
\text { Payment Instruments }\end{array}$} & Non-debit card users & Individuals who do not use on a monthly basis a debit card to make payments. \\
\hline & Debit card users & Individuals who use on a monthly basis a debit card to make payments. \\
\hline & Non-credit card users & Individuals who do not use on a monthly basis a credit card to make payments. \\
\hline & Credit card users & Individuals who use on a monthly basis a credit card to make payments. \\
\hline \multirow{3}{*}{$\begin{array}{l}\text { Adoption of Non-Bank } \\
\text { Payment Instruments }\end{array}$} & No digital users & $\begin{array}{l}\text { Individuals who are outside of the digitalization process (i.e., who have no access to the } \\
\text { internet). }\end{array}$ \\
\hline & $\begin{array}{l}\text { Non-users of non- } \\
\text { banking payment } \\
\text { methods }\end{array}$ & $\begin{array}{l}\text { Individuals who do not use at least once a month a payment method which is provided by a } \\
\text { non-bank institution (e.g. Amazon Pay, PayPal, Google Wallet, Apple Pay, etc.) were } \\
\text { classified as non-users of non-bank payment instruments. }\end{array}$ \\
\hline & $\begin{array}{l}\text { Users of non-banking } \\
\text { payment methods }\end{array}$ & $\begin{array}{l}\text { Individuals who use at least once a month a payment method which is provided by a non-bank } \\
\text { institution (e.g. Amazon Pay, PayPal, Google Wallet, Apple Pay, etc.) were classified as non- } \\
\text { users of non-bank payment instruments. }\end{array}$ \\
\hline
\end{tabular}

S4 Appendix. Alternative models performance in terms of predictive accuracy

\begin{tabular}{|c|c|c|c|c|c|c|c|}
\hline & & \multicolumn{6}{|c|}{ Out-of-sample accuracy (70/30\% split) } \\
\hline & & $\begin{array}{l}\text { Adoption of } \\
\text { online } \\
\text { banking }\end{array}$ & $\begin{array}{c}\text { Diversity of } \\
\text { digital use: } \\
\text { online banking }\end{array}$ & $\begin{array}{c}\text { Diversity of } \\
\text { digital use: } \\
\text { mobile } \\
\text { banking }\end{array}$ & Debit card & $\begin{array}{l}\text { Credit } \\
\text { card }\end{array}$ & $\begin{array}{c}\text { Adoption of } \\
\text { Non-bank } \\
\text { payment } \\
\text { methods }\end{array}$ \\
\hline \multicolumn{2}{|c|}{ Random forest } & $88.41 \%$ & $70.11 \%$ & $70.01 \%$ & $85.00 \%$ & $74.89 \%$ & $76.14 \%$ \\
\hline \multicolumn{2}{|c|}{ Extreme Gradient Boosting } & $84.99 \%$ & $68.82 \%$ & $67.85 \%$ & $84.79 \%$ & $73.51 \%$ & $75.91 \%$ \\
\hline \multirow{3}{*}{$\begin{array}{l}\text { K-Nearest } \\
\text { Neighbor }\end{array}$} & Euclidean & $84.92 \%$ & $63.41 \%$ & $63.97 \%$ & $80.60 \%$ & $64.75 \%$ & $74.94 \%$ \\
\hline & Manhattan & $82.71 \%$ & $60.53 \%$ & $62.97 \%$ & $80.27 \%$ & $65.96 \%$ & $72.73 \%$ \\
\hline & Chebyshev & $65.85 \%$ & $50.55 \%$ & $50.67 \%$ & $79.71 \%$ & $58.76 \%$ & $66.19 \%$ \\
\hline \multirow{4}{*}{$\begin{array}{l}\text { Supportive } \\
\text { Vector Machine } \\
\text { (SVM) }\end{array}$} & Linear & $83.54 \%$ & $69.00 \%$ & $66.70 \%$ & $82.75 \%$ & $71.14 \%$ & $74.15 \%$ \\
\hline & Radial & $84.58 \%$ & $67.36 \%$ & $66.27 \%$ & $82.11 \%$ & $72.63 \%$ & $74.48 \%$ \\
\hline & Sigmoid & $83.43 \%$ & $68.89 \%$ & $66.16 \%$ & $79.23 \%$ & $65.60 \%$ & $73.49 \%$ \\
\hline & Polynomial & $80.32 \%$ & $58.60 \%$ & $61.56 \%$ & $79.98 \%$ & $67.84 \%$ & $72.95 \%$ \\
\hline \multirow{8}{*}{$\begin{array}{l}\text { Bayesian } \\
\text { Networks }\end{array}$} & Naive Bayes & $58.52 \%$ & $39.55 \%$ & $43.52 \%$ & $67.16 \%$ & $56.25 \%$ & $53.30 \%$ \\
\hline & Tan HSCP & $58.98 \%$ & $41.48 \%$ & $43.18 \%$ & $65.91 \%$ & $55.68 \%$ & $52.61 \%$ \\
\hline & Tan CL & $34.55 \%$ & $25.34 \%$ & $28.64 \%$ & $53.86 \%$ & $49.09 \%$ & $35.23 \%$ \\
\hline & Tan HC & $57.39 \%$ & $40.45 \%$ & $41.02 \%$ & $65.57 \%$ & $57.95 \%$ & $52.16 \%$ \\
\hline & AODE & $58.07 \%$ & $42.39 \%$ & $42.95 \%$ & $65.68 \%$ & $57.50 \%$ & $53.86 \%$ \\
\hline & KBD & $56.82 \%$ & $39.89 \%$ & $44.66 \%$ & $66.70 \%$ & $58.30 \%$ & $51.59 \%$ \\
\hline & FSSJ & $86.48 \%$ & $66.36 \%$ & $63.98 \%$ & $84.43 \%$ & $72.16 \%$ & $70.91 \%$ \\
\hline & BESJ & $81.14 \%$ & $66.00 \%$ & $60.91 \%$ & $75.00 \%$ & $64.55 \%$ & $66.93 \%$ \\
\hline \multirow{10}{*}{$\begin{array}{l}\text { Artificial Neural } \\
\text { Networks: } \\
\text { Extreme learning } \\
\text { machine }\end{array}$} & Sigmoid & $58.48 \%$ & $40.24 \%$ & $45.12 \%$ & $79.82 \%$ & $54.55 \%$ & $64.86 \%$ \\
\hline & Radial Basis & $41.22 \%$ & $28.60 \%$ & $29.49 \%$ & $59.20 \%$ & $48.78 \%$ & $45.68 \%$ \\
\hline & Sine & $37.65 \%$ & $26.50 \%$ & $30.27 \%$ & $52.99 \%$ & $51.77 \%$ & $37.92 \%$ \\
\hline & Hard-Limit & $56.29 \%$ & $39.58 \%$ & $44.24 \%$ & $79.82 \%$ & $54.10 \%$ & $64.86 \%$ \\
\hline & Symm. Hard-Limit & $58.48 \%$ & $39.91 \%$ & $44.35 \%$ & $78.38 \%$ & $54.32 \%$ & $61.31 \%$ \\
\hline & Satlins & $73.49 \%$ & $60.31 \%$ & $61.86 \%$ & $82.59 \%$ & $69.73 \%$ & $73.17 \%$ \\
\hline & Tan-Sigmoid & $58.48 \%$ & $40.24 \%$ & $45.79 \%$ & $77.83 \%$ & $54.21 \%$ & $64.86 \%$ \\
\hline & Triangular Basis & $82.18 \%$ & $65.63 \%$ & $67.96 \%$ & $84.15 \%$ & $74.61 \%$ & $76.05 \%$ \\
\hline & Rectifier Linear Unit & $82.18 \%$ & $65.41 \%$ & $61.75 \%$ & $83.26 \%$ & $74.84 \%$ & $75.84 \%$ \\
\hline & Linear Function & $82.44 \%$ & $65.96 \%$ & $67.74 \%$ & $84.04 \%$ & $74.72 \%$ & $75.94 \%$ \\
\hline \multicolumn{2}{|l|}{ Logit } & $79.27 \%$ & $55.01 \%$ & $59.57 \%$ & $84.23 \%$ & $70.62 \%$ & $73.46 \%$ \\
\hline
\end{tabular}

S4 Appendix reports the predicted accuracy for all the models (machine learning algorithm and logit) employed in examining the digitalization of bank customers. 
S5 Appendix. Features importance by the Extreme Gradient Booting algorithm
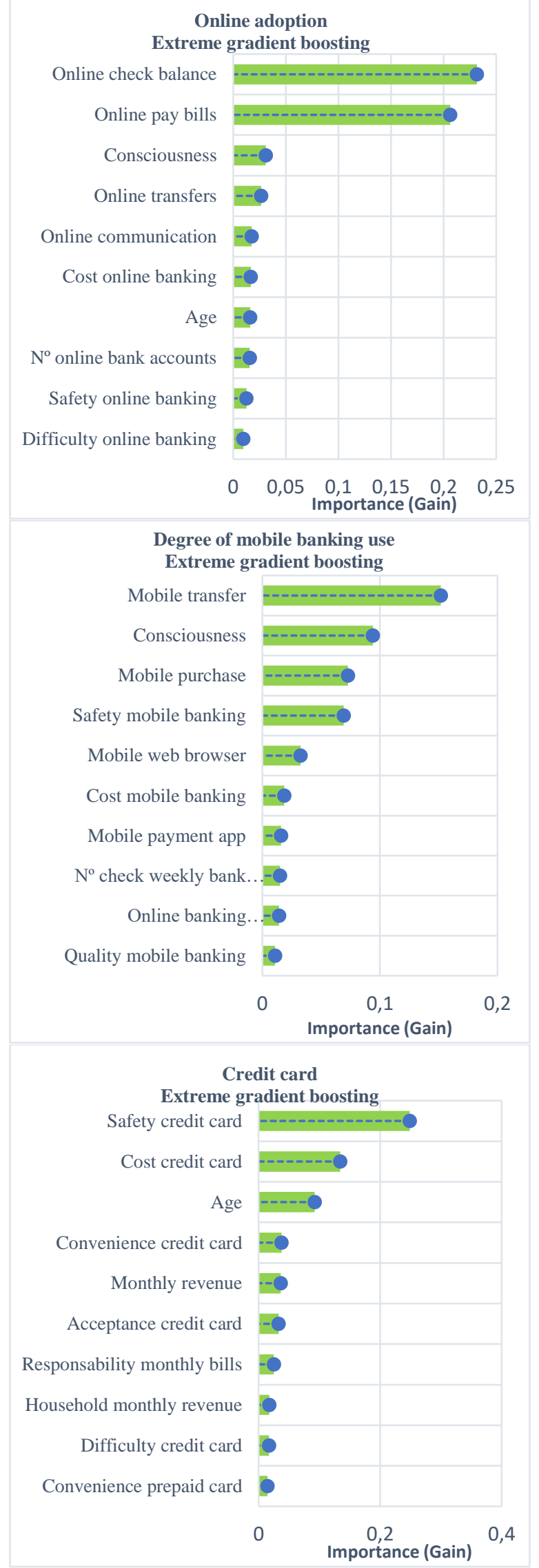

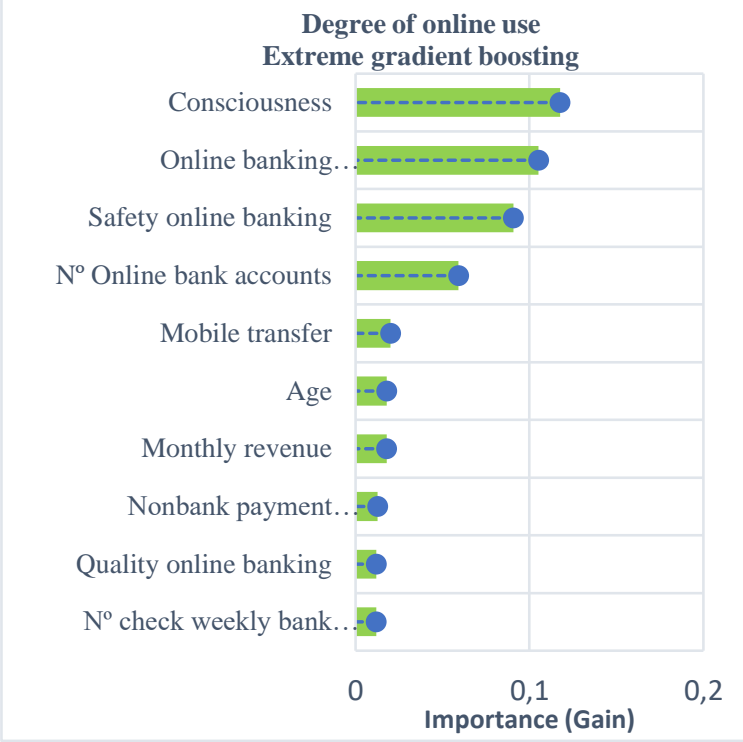

\section{Debit card}

Extreme gradient boosting
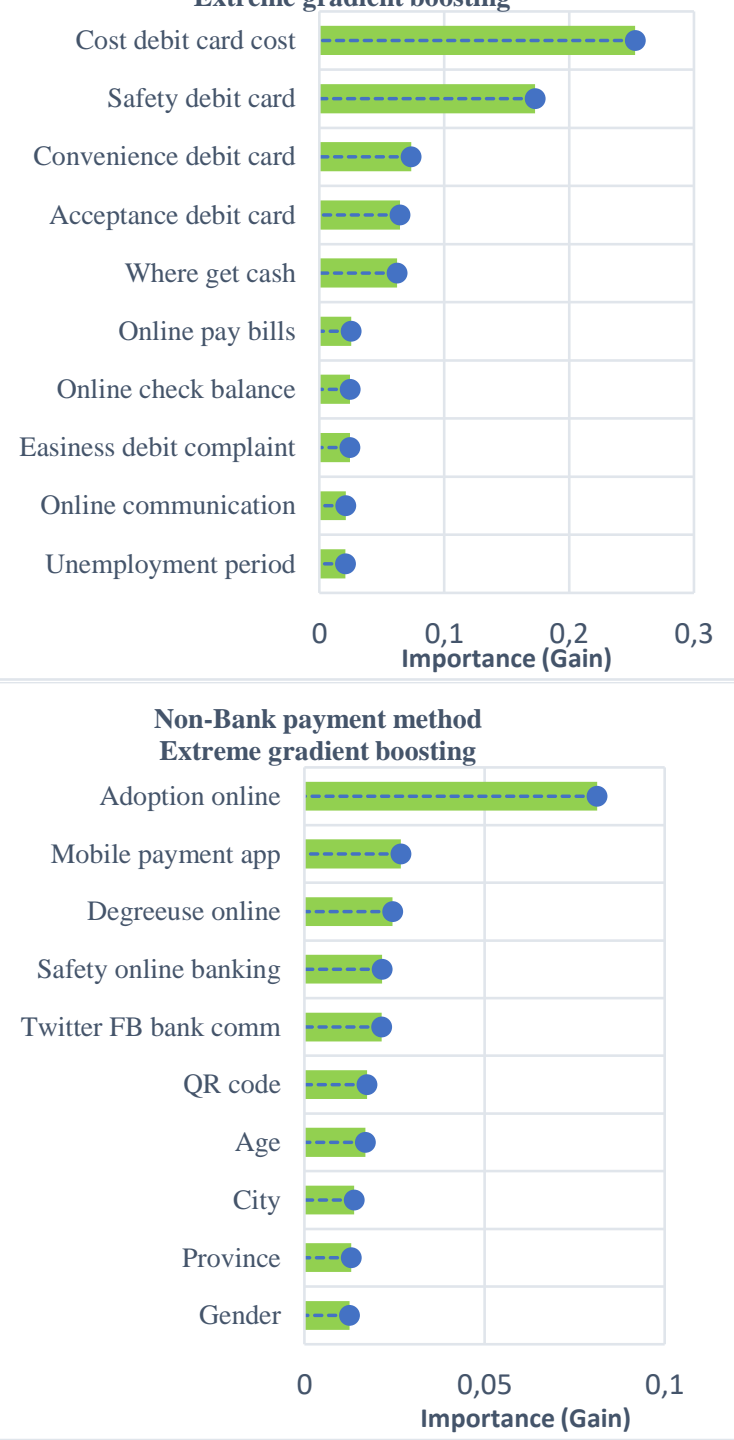

These figures provide the most important features predicting bank customers' digitalization based on the Extreme gradient boosting algorithm. The relative importance of each feature is computed using the relative contribution of the corresponding feature to the model calculated by taking each feature's contribution for each tree in the model (Gain). A higher score suggests the feature is more important in the boosted tree prediction. 
S6 Appendix. Bayesian Networks.

Figure S6.1 Bayesian Network: Adoption of digital banking

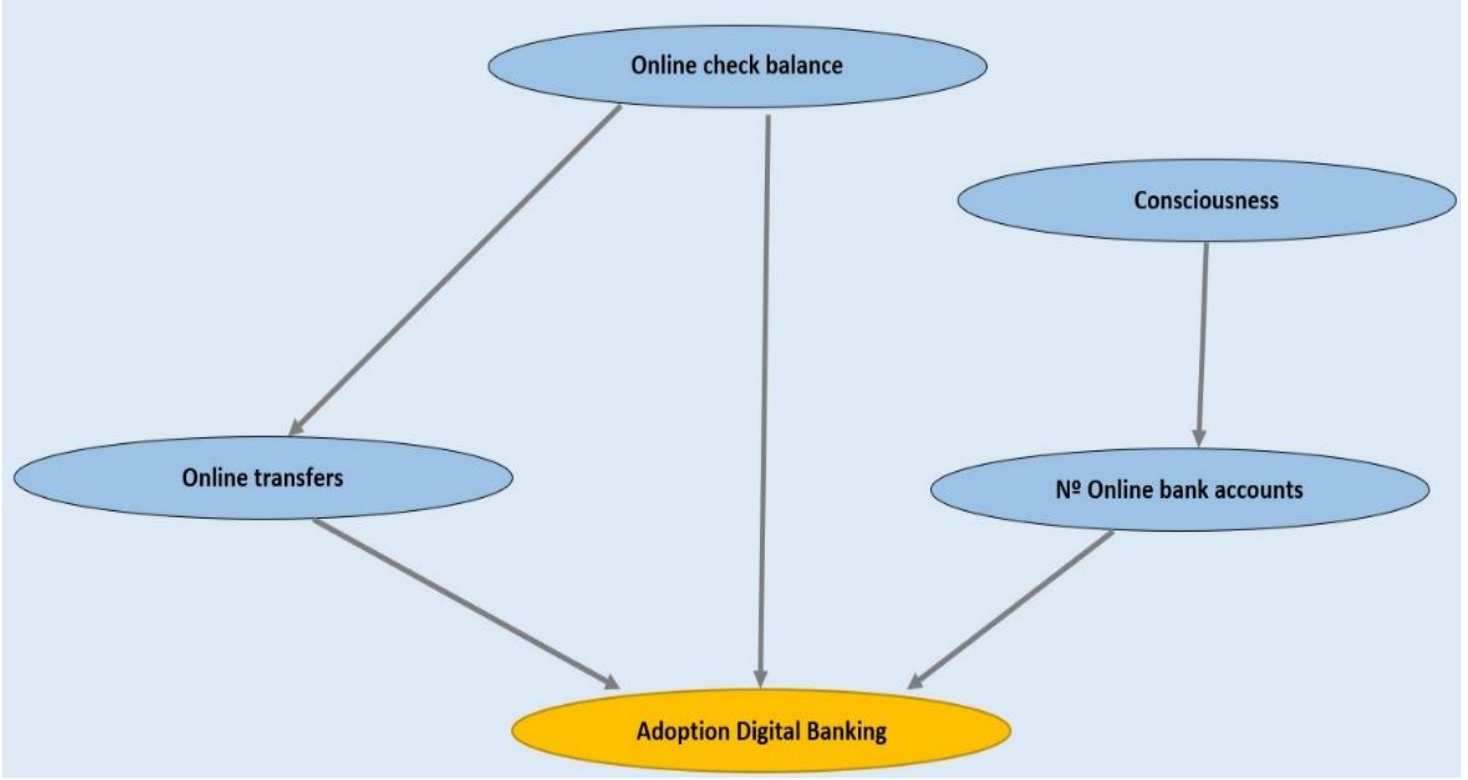

Figure S6.2 Bayesian Network: Diversity of use - Online banking

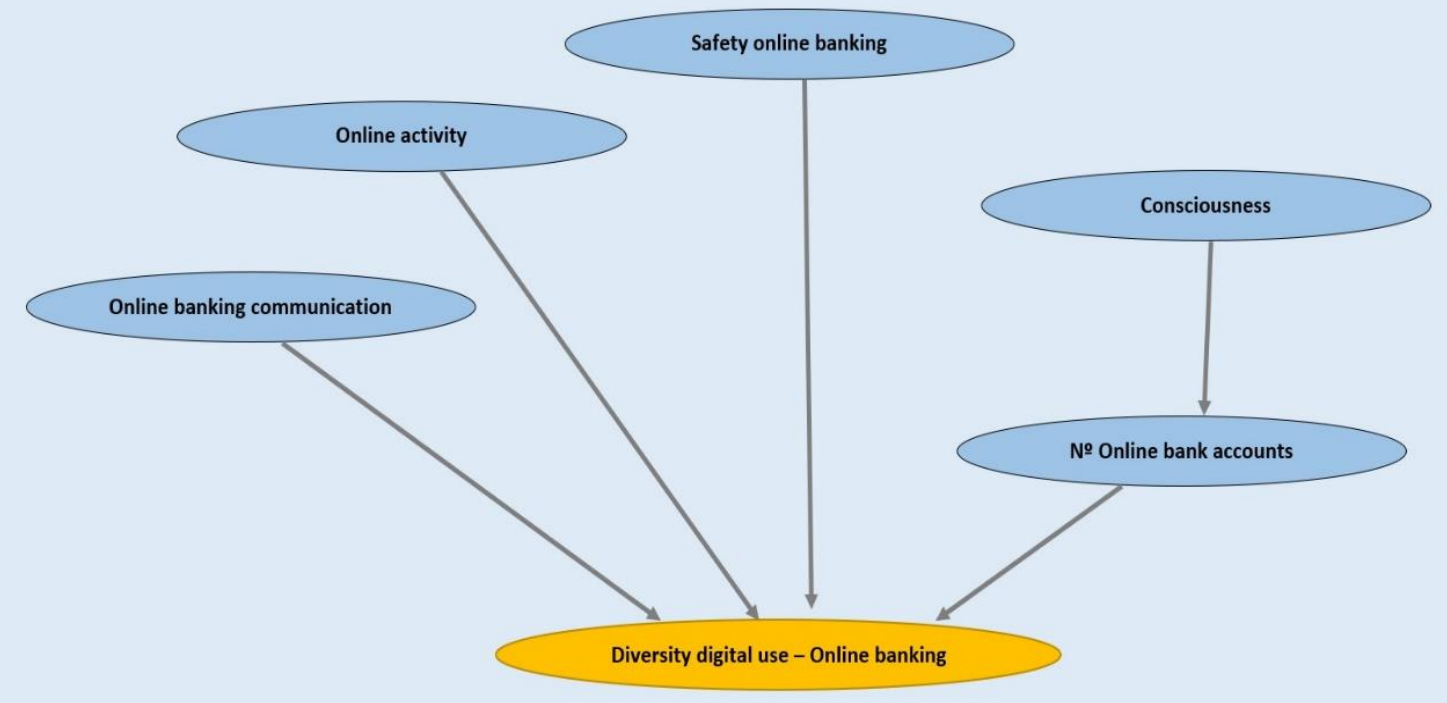

Figure S6.3 Bayesian Network: Diversity of use - Mobile banking

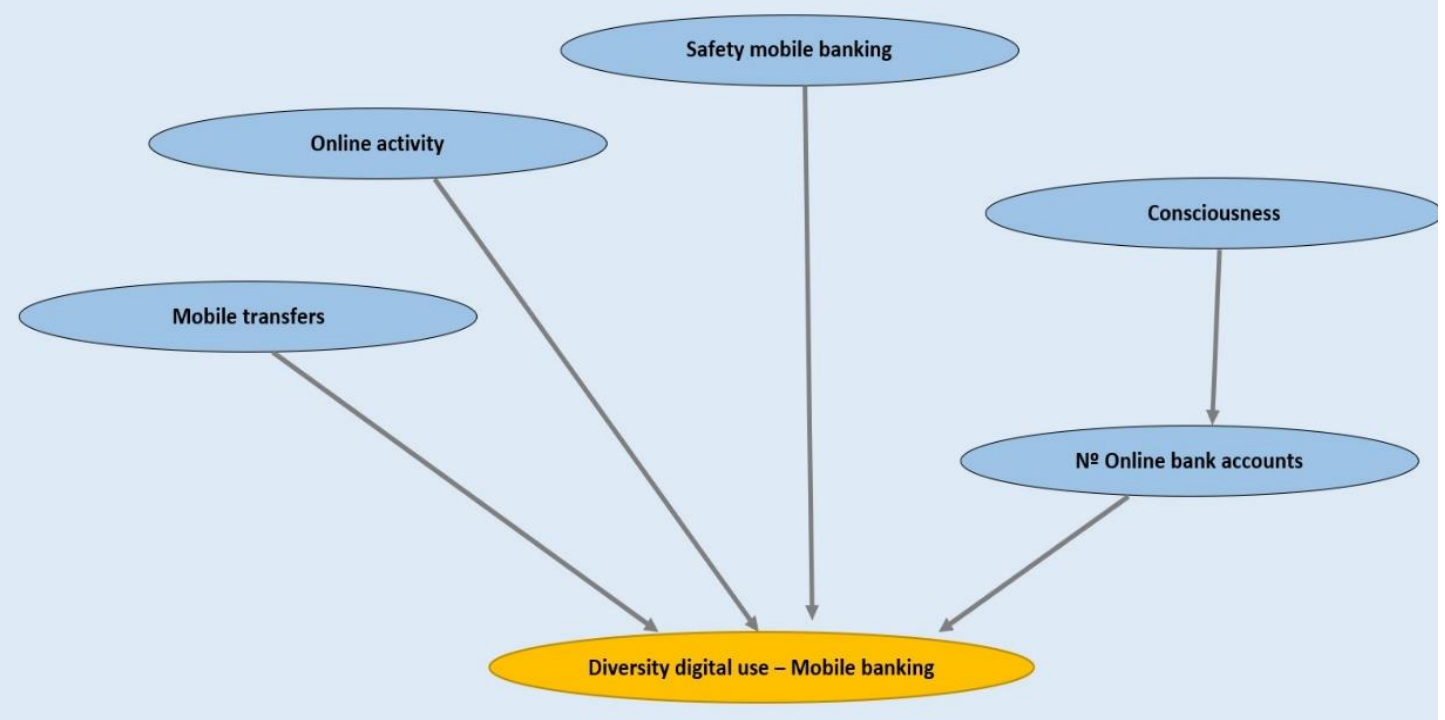


Figure S6.4 Bayesian Network: Debit card use

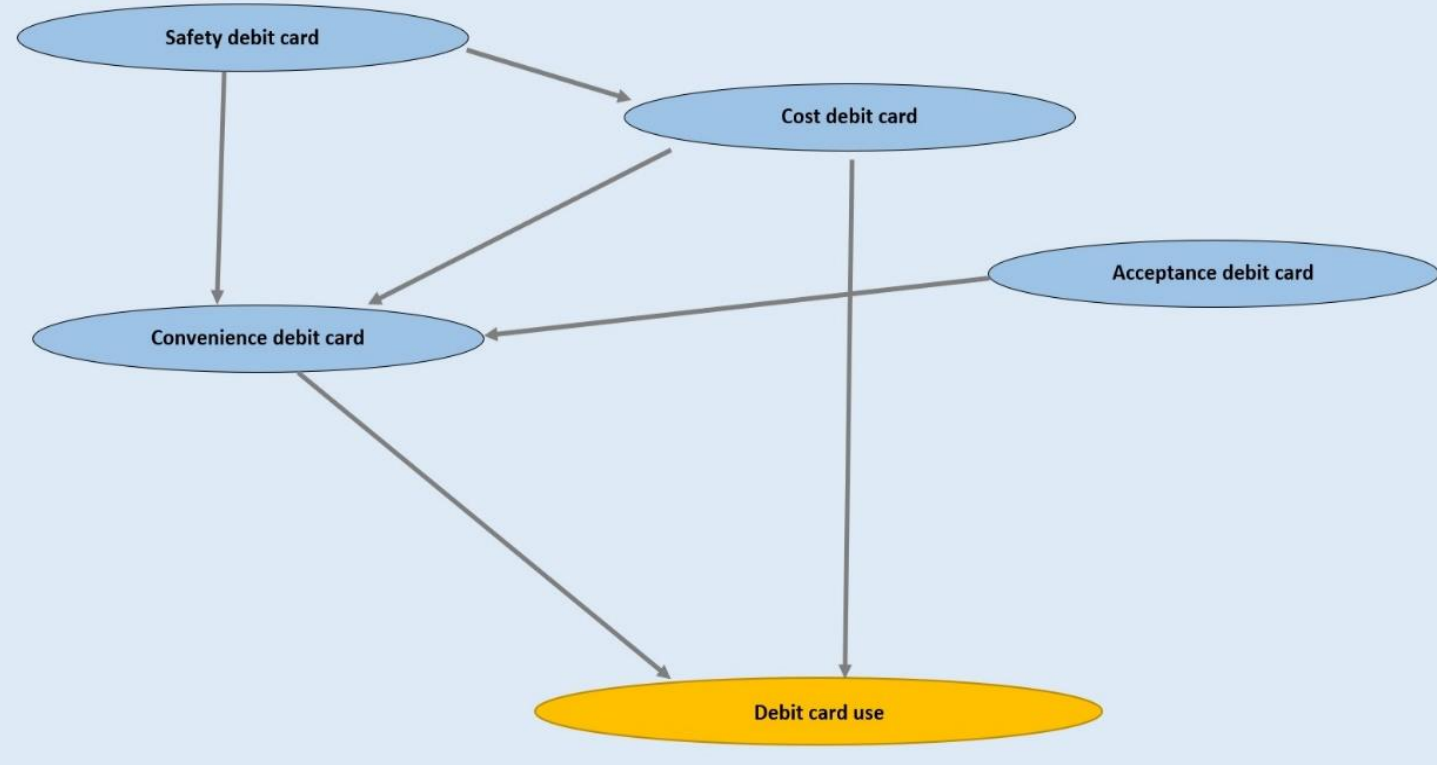

\section{Figure S6.5 Bayesian Network: Credit card use}

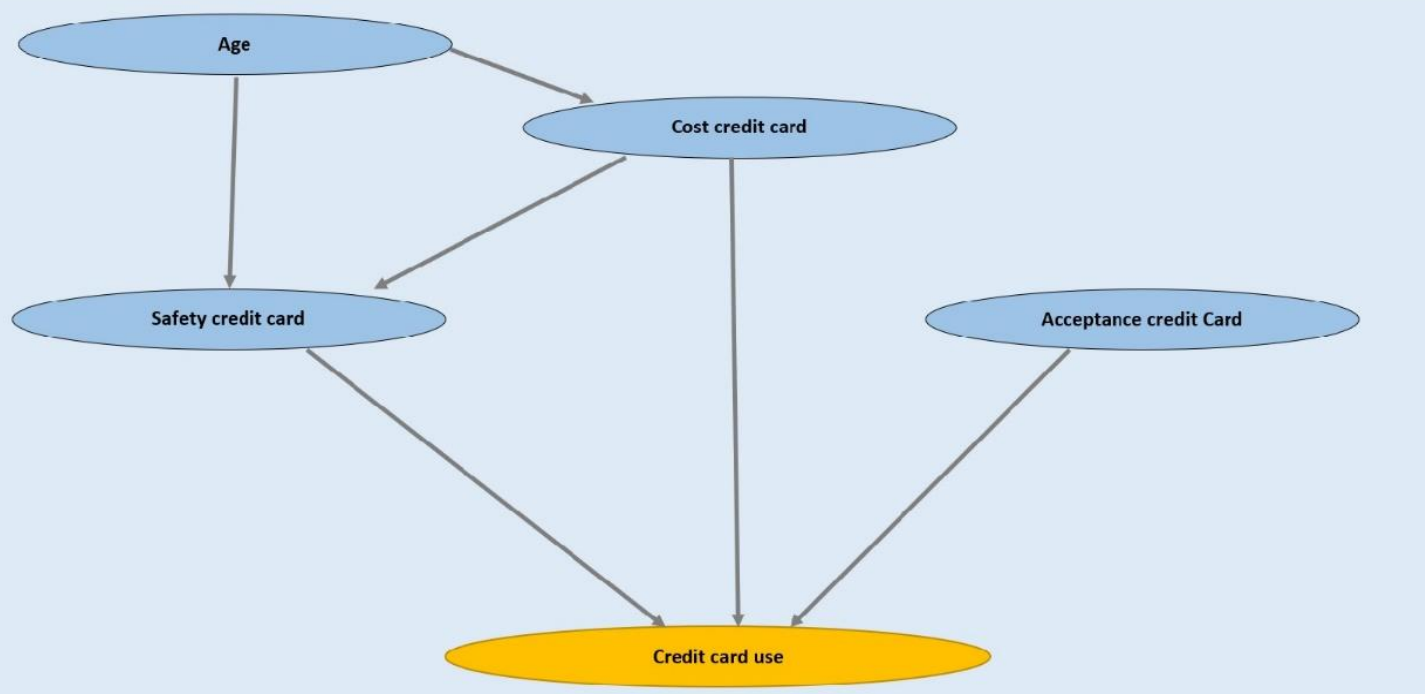

Figure S6.5 Bayesian Network: Use of non-bank payment instruments

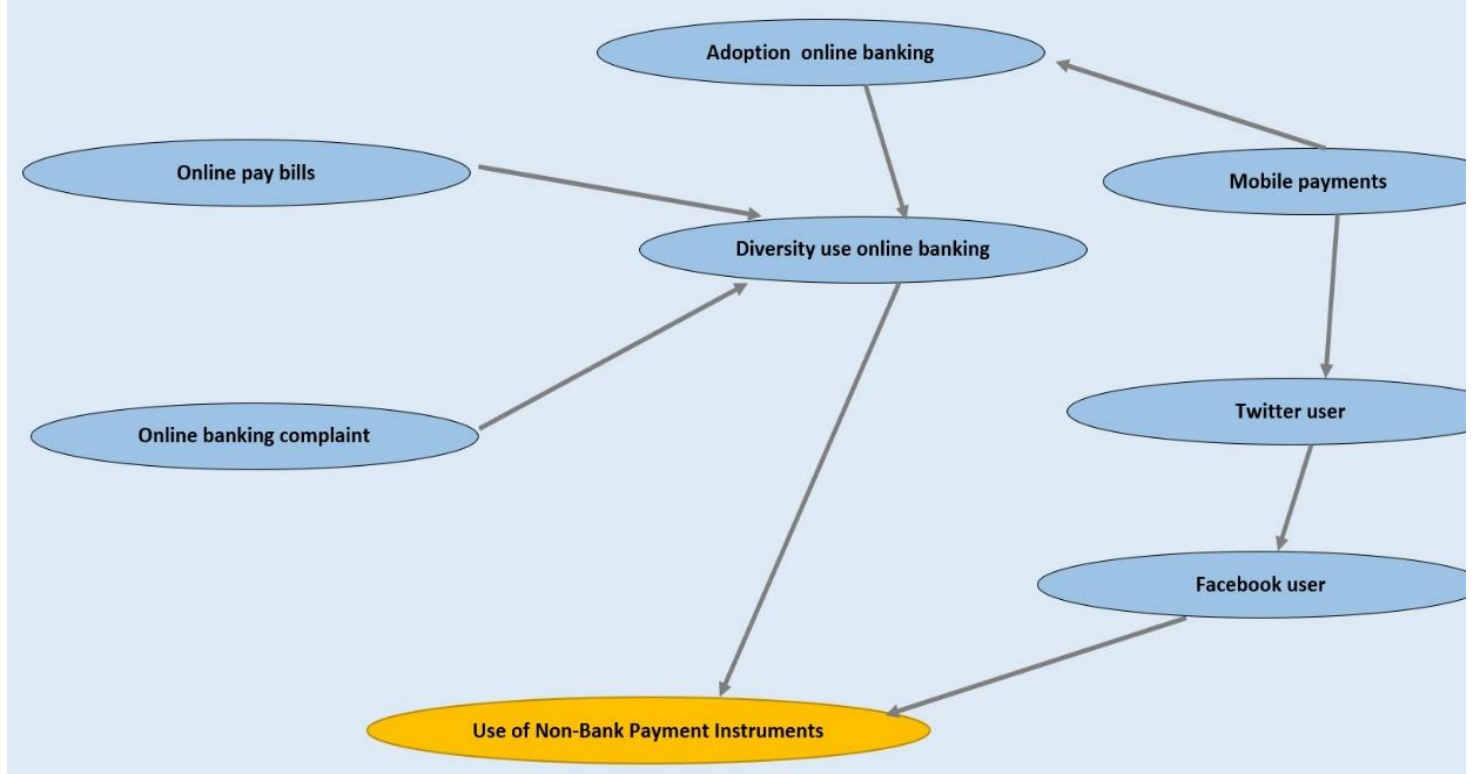

These figures plot the Bayesian network, based on the hill-climbing algorithm, for the subset of features with the largest discriminant power for each of the dimensions considered. 